\title{
THE ARMENDARIZ MODULE AND ITS APPLICATION TO THE IKEDA-NAKAYAMA MODULE
}

\section{TAMER KOŞAN}

Received 21 December 2005; Revised 5 July 2006; Accepted 5 September 2006

A ring $R$ is called a right Ikeda-Nakayama (for short IN-ring) if the left annihilator of the intersection of any two right ideals is the sum of the left annihilators, that is, if $\ell(I \cap$ $J)=\ell(I)+\ell(J)$ for all right ideals $I$ and $J$ of $R . R$ is called Armendariz ring if whenever polynomials $f(x)=a_{0}+a_{1} x+\cdots+a_{m} x^{m}, g(x)=b_{0}+b_{1} x+\cdots+b_{n} x^{n} \in R[x]$ satisfy $f(x) g(x)=0$, then $a_{i} b_{j}=0$ for each $i, j$. In this paper, we show that if $R[x]$ is a right IN-ring, then $R$ is a right IN-ring in case $R$ is an Armendariz ring.

Copyright (c) 2006 Hindawi Publishing Corporation. All rights reserved.

\section{Introduction}

Throughout this work, all rings will be associative with identity. Let $R$ be a ring. A right (or left) annihilator of a subset $U$ of $R$ is defined by $r_{R}(U)=\{a \in R: U a=0\}\left(\right.$ or $\ell_{R}(U)=$ $\{a \in R: a U=0\})$.

Recall that, a ring $R$ is called a right Ikeda-Nakayama ring if the left annihilator of the intersection of any two right ideals is the sum of the left annihilators, that is, if $\ell(I \cap J)=$ $\ell(I)+\ell(J)$ for all right ideals $I$ and $J$ of $R$ (cf. [6]). Let ${ }_{S} M_{R}$ be an $(S, R)$-bimodule. Extend the notion of an IN-ring to module such as $\ell_{S}(A \cap B)=\ell_{S}(A)+\ell_{S}(B)$ for any submodules $A, B$ of $M_{R}$ (cf. [10]).

For a module $M_{R}$, let $M[x]$ be the set of all formal polynomials in indeterminate $x$ with coefficients from $M$. Then $M[x]$ becomes a right $R[x]$-module under usual addition and multiplication of polynomials.

We prove that if ${ }_{S[x]} M[x]_{R[x]}$-bimodule and $U$ and $V$ are $R[x]$-submodules of $M[x]_{R[x]}$, then for any $t(x) \in \ell_{S[x]}(U \cap V)$, every $U+V \stackrel{\alpha_{t(x)}}{\longrightarrow} M[x]$ extends commutatively to $M[x]$ by $\lambda(s(x))$ for some $s(x) \in S[x]$, where $\lambda: S[x] \rightarrow \operatorname{End}\left(M[x]_{R[x]}\right)$ if and only if $M[x]$ is an IN-module.

Following [1], $R$ is called Armendariz ring if whenever polynomials $f(x)=a_{0}+a_{1} x+$ $\cdots+a_{m} x^{m}$ and $g(x)=b_{0}+b_{1} x+\cdots+b_{n} x^{n} \in R[x]$ satisfy $f(x) g(x)=0$, then $a_{i} b_{j}=0$ for each $i, j$. 
A module $M$ is called $\alpha$-Armendariz if

(i) for any $m \in M$ and $a \in R, m a=0$ if and only if $m \alpha(a)=0$;

(ii) for any $m(x)=\sum_{i=0}^{n} m_{i} x^{i} \in M[x]$ and $f(x)=\sum_{j=0}^{s} a_{j} x^{j} \in R[x], m(x) f(x)=0$ implies $m_{i} a_{j}=0$ for each $i, j$ (cf. $[8,9]$ ).

In [5, Proposition 3.1], Hirano showed that $R$ is Armendariz ring if and only if $r \operatorname{Ann}_{R}\left(2^{R}\right) \rightarrow r \operatorname{Ann}_{R}\left(2^{R[x]}\right) ; A \rightarrow A R[x]$ is bijective, where $r \operatorname{Ann}_{R}\left(2^{R}\right)=\left\{r_{R}(U): U \subseteq R\right\}$. Using this proposition, in this paper, it is shown that if $R[x]$ is a right IN-ring, then $R$ is a right IN-ring, in case $R$ is an Armendariz ring.

\section{Ikeda-Nakayama modules}

Let $S[x]$ and $R[x]$ be the polynomial rings over rings $S$ and $R$ and, for a module ${ }_{S} M_{R}$, let $M[x]$ be the set of all formal polynomials in indeterminate $x$ with coefficients from $M$. Then $M[x]$ becomes an $(S[x], R[x])$-bimodule under usual addition and multiplication of polynomials. Extend the notion of an IN-ring to module such as the following.

Definition 2.1. Recall that $M[x]$ is called an Ikeda-Nakayama module (IN-module) if

$$
\ell_{S[x]}(U \cap V)=\ell_{S[x]}(U)+\ell_{S[x]}(V)
$$

for any $R[x]$-submodules $U$ and $V$ of $M[x]_{R[x]}$. Such modules and rings were studied by many authors (cf. $[4,6,10]$ ). Professor Harmanci asked (private communication) for a description modules $M$ (rings $R$ ) such that $M[x](R[x])$ are Ikeda-Nakayama modules (right Ikeda-Nakayama rings), respectively.

Note that there is a canonical ring homomorphism $\lambda: S[x] \rightarrow \operatorname{End}\left(M[x]_{R[x]}\right)$ given by $\lambda(s(x))(m(x))=s(x) m(x)$ for $m(x) \in M[x]$ and $s(x) \in S[x]$.

Let $U$ and $V$ be $R[x]$-submodules of $M[x]$. Then, for any $t(x) \in \ell_{S[x]}(U \cap V), \alpha_{t(x)}$ : $U+V \rightarrow M[x], u+v \rightarrow t(x) u$ is well defined, where $u \in U$ and $v \in V$.

Lemma 2.2. Let ${ }_{S[x]} M[x]_{R[x]}$-bimodule and $U$ and $V$ be $R[x]$-submodules of $M[x]_{R[x]}$. Then, for any $t(x) \in \ell_{S[x]}(U \cap V)$, every $U+V \stackrel{\alpha_{t(x)}}{\longrightarrow} M[x]$ extends commutatively to $M[x]$ by $\lambda(s(x))$ for some $s(x) \in S[x]$ if and only if $M[x]$ is an IN-module.

In particular, if $U \cap V=0$, then every $U+V \stackrel{\alpha_{1}}{\longrightarrow} M[x]$ extends commutatively to $M[x]$ by $\lambda(s(x))$ for some $s(x) \in S[x]$ if and only if $S[x]=\ell_{S[x]}(U)+\ell_{S[x]}(V)$.

Proof. Let $t(x) \in \ell_{S[x]}(U \cap V)$. Then $\alpha_{t(x)}: U+V \rightarrow M[x], u+v \rightarrow t(x) u$ is a well defined $R[x]$-module homomorphism, where $u \in U$ and $v \in V$. By assumption, there exists $s(x) \in S[x]$ such that $\lambda(s(x))$ extends to $\alpha_{t(x)}$. Thus, for all $u \in U$ and $v \in V, t(x) u=$ $\alpha_{t(x)}(u+v)=\lambda(s(x))(u+v)=s(x)(u+v)$ and so $(t(x)-s(x)) u+(-s(x)) v=0$. It follows that $t(x)-s(x) \in \ell_{S[x]}(U)$ and $-s(x) \in \ell_{S[x]}(V)$. Hence $t(x)=(t(x)-s(x))+(-s(x)) \in$ $\ell_{S[x]}(U)+\ell_{S[x]}(V)$. The other inclusion is clear.

For converse, assume that $M[x]$ is an IN-module and, for any $t(x) \in \ell_{S[x]}(U \cap V)$, $\alpha_{t(x)}: U+V \rightarrow M[x]$ defined as above. For $a(x) \in \ell_{S[x]}(U)$ and $b(x) \in \ell_{S[x]}(V)$, write $t(x)=a(x)+b(x)$. Then, for all $u \in U$ and $v \in V, \alpha_{t(x)}(u+v)=t(x) u=(a(x)+b(x)) u=$ $a(x) u+b(x) u=0+b(x) u=b(x) u=b(x) u+b(x) v=b(x)(u+v)=\lambda(b(x)(u+v))$.

As a result of Lemma 2.2, we have the following proposition. 
Proposition 2.3. Let $R[x]$ be the set of all polynomials in indeterminate $x$ with coefficients from $R$. If $I$ and $J$ are right ideals of $R[x]$ such that every $R[x]$-linear map $I+J \rightarrow R[x]$ extends to $R[x]$, then

$$
\ell_{R[x]}(I \cap J)=\ell_{R[x]}(I)+\ell_{R[x]}(J) .
$$

In particular, this holds if $I+J=R[x]$, in which case $\ell_{R[x]}(I \cap J)=\ell_{R[x]}(I) \oplus \ell_{R[x]}(J)$.

Let $N$ be an $R[x]$-submodule of $M[x]$ and $N_{C}=\left\{m_{i} \in M: \exists n \in N\right.$ with $n=m_{0}+$ $\left.m_{1} x+\cdots+m_{t} x^{t}\right\}$.

Theorem 2.4. Let $M$ be an Ikeda-Nakayama module and let $N$ and $K$ be $R[x]$-submodules of $M[x]$ such that $\ell_{S}\left((N \cap K)_{C}\right)=\ell_{S}\left(N_{C} \cap K_{C}\right)$. Then $M[x]$ is an IN-module.

Proof. Let $U$ and $V$ be $R[x]$-submodules of $M[x]$. Let $t(x) \in \ell_{S[x]}(U \cap V)$. Then $\alpha_{t(x)}$ : $U+V \rightarrow M[x], u+v \rightarrow t(x) u$ is a well defined $R[x]$-homomorphism, where $u \in U$ and $v \in V$. Similarly, for all $t \in \ell_{S}\left(U_{C} \cap V_{C}\right)$, the $\alpha_{t}: U_{C}+V_{C} \rightarrow M, u^{\prime}+v^{\prime} \rightarrow t u^{\prime}$ is a well defined $R$-homomorphism, where $u^{\prime} \in U_{C}$ and $v^{\prime} \in V_{C}$. Since $M$ is an IN-module, we have $\left.\ell_{S}(U \cap V)_{C}\right)=\ell_{S}\left(N_{C} \cap K_{C}\right)=\ell_{S}\left(U_{C}\right)+\ell_{S}\left(V_{C}\right)$ by assumption and definition. Hence there exists a homomorphism $h_{t}: M \rightarrow M$ such that $h_{t} i=\alpha_{t}$, where $i: U_{C}+V_{C} \rightarrow M$ is the inclusion map by [10, Lemma 1]. We define $h^{\prime}: M[x] \rightarrow M[x]$ such that $h_{t}^{\prime}\left(k_{0}+k_{1} x+\right.$ $\left.\cdots+k_{n} x^{n}\right)=h_{t}\left(k_{0}\right)+h_{t}\left(k_{1}\right) x+\cdots+h_{t}\left(k_{n}\right) x^{n}$. It is clear that $h_{t}^{\prime}$ is well defined. Let $t(x)=$ $t_{0}+t_{1} x+t_{2} x^{2}+\cdots+t_{n} x^{n} \in \ell_{S[x]}(U \cap V)$. Then $t_{0}, t_{1}, \ldots, t_{n} \in \ell_{S}\left((U \cap V)_{C}\right)=\ell_{S}\left(U_{C}\right)+$ $\ell_{S}\left(V_{C}\right)$. For each $t_{j}, \alpha_{t_{j}}: U_{C}+V_{C} \rightarrow M, u^{\prime}+v^{\prime} \rightarrow t u^{\prime}$ is a well defined $R$-homo-morphism, and then we define a map $h_{t_{j}}: M \rightarrow M$ such that $h_{t_{j}} i=\alpha_{t_{j}}$, where $i: U_{C}+V_{C} \rightarrow M$ is the inclusion map. We extend it by defining $h_{t_{j}}^{\prime}: M[x] \rightarrow M[x]$ such that, for $j=0,1,2, \ldots, n$, $h_{j}^{\prime}\left(k_{0}+k_{1} x+\cdots+k_{n} x^{n}\right)=\left(h_{t_{j}}\left(k_{0}\right)+h_{t_{j}}\left(k_{1}\right) x+\cdots+h_{t_{j}}\left(k_{n}\right) x^{n}\right) x^{j}$.

To complete the proof, we show that $h i=\alpha_{t(x)}$, where $i^{\prime}: U+V \rightarrow M[x]$ is the inclusion map. Let $h=\sum_{j=0}^{n} h_{j}^{\prime}$ and $u=u_{0}+u_{1} x+\cdots+u_{r} x^{r} \in U$ and $v(x)=v_{0}+v_{1} x+$ $\cdots+v_{s} x^{s} \in V$. Then $u_{0}, u_{1}, \ldots, u_{r} \in U_{C}$ and $v_{0}, v_{1}, \ldots, v_{s} \in V_{C}$. So $h_{j}^{\prime}(u+v)=\left(h_{t_{j}}\left(u_{0}\right)+\right.$ $\left.h_{t_{j}}\left(u_{1}\right) x+\cdots+h_{t_{j}}\left(u_{r}\right) x^{r}\right) x^{j}=t_{j} x^{j}\left(u_{0}+u_{1} x+\cdots+u_{r} x^{r}\right)$ and $h(u+v)=\sum_{j=0}^{n} h_{j}^{\prime}(u+v)=$ $t(x)(u+v)$. Hence $M[x]$ is an IN-module by Lemma 2.2 .

Let $\alpha$ be an endomorphism of $R$, that is, $\alpha$ is a ring homomorphism from $R$ to $R$ with $\alpha(1)=1$. Following [9], a module $M$ is called $\alpha$-Armendariz if

(1) for any $m \in M$ and $a \in R, m a=0$ if and only if $m \alpha(a)=0$;

(2) for any $m(x)=\sum_{i=0}^{n} m_{i} x^{i} \in M[x]$ and $f(x)=\sum_{j=0}^{s} a_{j} x^{j} \in R[x], m(x) f(x)=0$ implies $m_{i} a_{j}=0$ for each $i, j$.

Note that 1-Armendariz module is called Armendariz module.

We denote $r \operatorname{Ann}_{R}\left(2^{M}\right)=\left\{r_{R}(U) \mid U \subseteq M\right\}$ and $\ell \operatorname{Ann}_{R}\left(2^{M}\right)=\left\{\ell_{R}(U) \mid U \subseteq M\right\}$. If $U$ is a subset of $M$, then $\ell_{R[x]}(U)=\ell_{R}(U)[x]$ and $r_{R[x]}(U)=r_{R}(U)[x]$. Hence we have the maps

$$
\Phi: r \operatorname{Ann}_{R}\left(2^{M}\right) \longrightarrow r \operatorname{Ann}_{R[x]}\left(2^{M[x]}\right)
$$


defined by $\Phi\left(r_{R}(U)\right)=r_{R[x]}(U)=r_{R}(U)[x]$ for every $r_{R}(U) \in r \operatorname{Ann}_{R}\left(2^{M}\right)$ and

$$
\Phi^{\prime}: \ell \operatorname{Ann}_{R}\left(2^{M}\right) \longrightarrow \ell \operatorname{Ann}_{R[x]}\left(2^{M[x]}\right)
$$

defined by $\Phi^{\prime}\left(\ell_{R}(U)\right)=\ell_{R[x]}(U)=\ell_{R}(U)[x]$ for every $\ell_{R}(U) \in \ell \operatorname{Ann}_{R}\left(2^{M}\right)$.

For a polynomial $m(x) \in M[x], C_{m(x)}$ denotes the set of coefficients of $m(x)$ and for a subset $V$ of $M[x], C_{V}$ denotes the set $\bigcup_{m(x) \in V} C_{m(x)}$. Then

$$
r_{R[x]}(V) \cap R=r_{R}(V)=r_{R}\left(C_{V}\right), \quad \ell_{R[x]}(V) \cap R=\ell_{R}(V)=\ell_{R}\left(C_{V}\right) .
$$

Hence we also have the maps

$$
\Psi: r \operatorname{Ann}_{R[x]}\left(2^{M[x]}\right) \longrightarrow r \operatorname{Ann}_{R}\left(2^{M}\right)
$$

defined by $\Psi\left(r_{R[x]}(V)\right)=r_{R[x]}(V) \cap R$ for every $r_{R[x]}(V) \in r \operatorname{Ann}_{R[x]}\left(2^{M[x]}\right)$ and

$$
\Psi^{\prime}: \ell \operatorname{Ann}_{R[x]}\left(2^{M[x]}\right) \longrightarrow \ell \operatorname{Ann}_{R}\left(2^{M}\right)
$$

defined by $\Psi^{\prime}\left(\ell_{R[x]}(V)\right)=\ell_{R[x]}(V) \cap R$ for every $\ell_{R[x]}(V) \in \ell \operatorname{Ann}_{R[x]}\left(2^{M[x]}\right)$.

Obviously $\Phi$ (or $\left.\Phi^{\prime}\right)$ is injective and $\Psi$ (or $\Psi^{\prime}$ ) is surjective. Also, $\Phi$ (or $\Phi^{\prime}$ ) is surjective if and only if $\Psi\left(\right.$ or $\left.\Psi^{\prime}\right)$ is injective and in this case $\Phi$ and $\Psi$ (or $\Phi^{\prime}$ and $\Psi^{\prime}$ ) are the inverses of each other.

Proposition 2.5. Let $M_{R}$ be a module. Then the following are equivalent.

(1) $M_{R}$ is an Armendariz module.

(2) The map $\Phi: r A n n_{R}\left(2^{M}\right) \rightarrow r A n n_{R[x]}\left(2^{M[x]}\right)$ defined by $\Phi\left(r_{R}(U)\right)=r_{R[x]}(U)=$ $r_{R}(U)[x]$, for every $r_{R}(U) \in r A n n_{R}\left(2^{M}\right)$, is bijective.

(3) The map $\Phi^{\prime}: \ell A n n_{R}\left(2^{M}\right) \rightarrow \ell A n n_{R[x]}\left(2^{M[x]}\right)$ defined by $\Phi^{\prime}\left(\ell_{R}(U)\right)=\ell_{R[x]}(U)=$ $\ell_{R}(U)[x]$, for every $\ell_{R}(U) \in \ell A n n_{R}\left(2^{M}\right)$, is bijective.

Proof. $(1) \Leftrightarrow(2)$. This is [3, Theorem 2.1].

$(1) \Rightarrow(3)$. Assume $M$ is an Armendariz module. Obviously $\Phi^{\prime}$ is injective. So it is enough to show $\Phi^{\prime}$ is surjective. Let $\ell_{R[x]}(V) \in \ell \operatorname{Ann}_{R[x]}\left(2^{M[x]}\right)$ for some $V \subseteq M[x]$. Then for $\ell_{R}\left(C_{V}\right) \in \ell \operatorname{Ann}_{R}\left(2^{M}\right), \Phi^{\prime}\left(\ell_{R}\left(C_{V}\right)\right)=\ell_{R[x]}\left(C_{V}\right)=\ell_{R[x]}(V)$. In fact, let $f(x) \in$ $\ell_{R[x]}\left(C_{V}\right)$, where $f(x)=a_{0}+a_{1} x+\cdots+a_{n} x^{n}$. Then $f(x) C_{V}=0$. Thus for all $m \in C_{V}$, $f(x) m=a_{0} m+a_{1} m x+\cdots+a_{n} m x^{n}=0$ and hence $a_{j} m=0$ for all $j$. Let $n(x)=n_{0}+$ $n_{1} x+\cdots+n_{t} x^{t} \in V$ be arbitrary. Then $f(x) n(x)=0$ since $n_{i} \in C_{V}$ for all $i$. Hence $f(x) \in$ $\ell_{R[x]}(V)$. Conversely, let $g(x)=b_{0}+b_{1} x+\cdots+b_{k} x^{k} \in \ell_{R[x]}(V)$. Then for all $m(x) \in V$, $g(x) m(x)=0$, where $m(x)=m_{0}+m_{1} x+\cdots+m_{l} x^{l} \in V$. Since $M_{R}$ is Armendariz, $b_{j} m_{i}=$ 0 for all $i$ and $j$. Hence $g(x) m_{i}=0$ for all $i$. So $g(x) \in \ell_{R[x]}\left(C_{V}\right)$ since $m(x) \in V$ is arbitrary. Consequently for each $\ell_{R[x]}(V) \in \ell \operatorname{Ann}_{R[x]}\left(2^{M[x]}\right)$ for some $V \subseteq M[x]$ there exists $\ell_{R}\left(C_{V}\right) \in \ell_{\operatorname{Ann}_{R}}\left(2^{M}\right)$ such that $\Phi^{\prime}\left(\ell_{R}\left(C_{V}\right)\right)=\ell_{R[x]}(V)$, and therefore $\Phi^{\prime}$ is surjective. 
(3) $\Rightarrow(1)$. Conversely, assume $f(x) m(x)=0$, where $m(x)=m_{0}+m_{1} x+\cdots+m_{t} x^{t} \in$ $M[x]$ and $f(x)=a_{0}+a_{1} x+\cdots+a_{k} x^{k} \in R[x]$. By hypothesis, $\ell_{R[x]}(m(x))=\ell_{R}(U)[x]$ for some $U \subseteq M$. Then $f(x) \in \ell_{R}(U)[x]$ and hence $a_{j} \in \ell_{R}(U)$ for all $j$. So $a_{j} \in \ell_{R}(U) \subseteq$ $\ell_{R}(U)[x]=\ell_{R[x]}(m(x))$ then $a_{j} m(x)=0$. Consequently, $a_{j} m_{i}=0$ for all $i$ and $j$. Therefore $M_{R}$ is an Armendariz module.

By Proposition 2.5, we can obtain [5, Proposition 3.1].

Proposition 2.6. Let $R$ be a ring. The following statements are equivalent.

(1) $R$ is Armendariz ring.

(2) $r A n n_{R}\left(2^{R}\right) \rightarrow r A n n_{R}\left(2^{R[x]}\right) ; A \rightarrow A R[x]$ is bijective, where $r A n n_{R}\left(2^{R}\right)=\left\{r_{R}(U)\right.$ : $U \subseteq R\}$.

(3) $\ell A n n_{R}\left(2^{R}\right) \rightarrow \ell A n n_{R}\left(2^{R[x]}\right) ; B \rightarrow R[x] B$ is bijective, where $\ell A n n_{R}\left(2^{R}\right)=\left\{\ell_{R}(U)\right.$ : $U \subseteq R\}$.

Now, we give the main result of this work.

Theorem 2.7. Let $R$ be an Armendariz ring. If $R[x]$ is a right IN-ring, then $R$ is a right IN-ring.

Proof. Let $I$ and $J$ be right ideals of $R$. Since $R$ is an Armendariz ring, we have $\ell_{R[x]}(I)=$ $\ell_{R}(I)[x]$ by Proposition 2.6, for every right ideal $I$ of $R$. Note that $\ell_{R[x]}(I)=\ell_{R[x]}(I[x])$. By assumption, $\ell_{R[x]}(I)+\ell_{R[x]}(J)=\ell_{R[x]}(I[x])+\ell_{R[x]}(J[x])=\ell_{R[x]}(I[x] \cap J[x])=\ell_{R[x]}((I \cap$ $J)[x])=\ell_{R[x]}(I \cap J)$. Then $\ell_{R}((I \cap J)[x])=\ell_{R}(I[x])+\ell_{R}(J[x])=\left(\ell_{R}(I)+\ell_{R}(J)\right)[x]$ implies that $\ell_{R}(I \cap J)=\ell_{R}(I)+\ell_{R}(J)$. So $R$ is a right IN-ring.

Example 2.8. (i) Since $\mathbb{Z}$ is an Armendariz ring, $\mathbb{Z}$ is a right $I N$-ring if and only if $\mathbb{Z}[x]$ is an IN-ring.

(ii) Let $R$ be a trivial extension of $\mathbb{Z}$ and the $\mathbb{Z}$-module $\mathbb{Z}_{2^{\infty}}$, that is, $R=\mathbb{Z} \oplus \mathbb{Z}_{2^{\infty}}$ with the following addition and multiplication:

$$
\begin{gathered}
(n, a)+(m, b)=(n+m, a+b) \\
(n, a)(m, b)=(n m, n b+m a) .
\end{gathered}
$$

Also $R$ is the subring $\left\{\left(\begin{array}{ll}a & n \\ 0 & a\end{array}\right): a \in \mathbb{Z}, n \in \mathbb{Z}_{2^{\infty}}\right\} . R$ is an IN-ring by [10]. As Lee and Zhou pointed out [8, Corollary 2.7], $R$ is an Armendariz ring. We consider the right ideals $I$ and $J$ of $R[x]$ :

$$
\begin{aligned}
& I=\left\{\left(\begin{array}{cc}
p x^{2} & u(x) \\
0 & p x^{2}
\end{array}\right): u(x) \in \mathbb{Z}_{2^{\infty}}, p \text { is prime }\right\}, \\
& J=\left\{\left(\begin{array}{cc}
q x+q x^{2} & 0 \\
0 & q x+q x^{2}
\end{array}\right): q \text { is prime and }(p, q)=1\right\} .
\end{aligned}
$$

Clearly, $\ell_{R[x]}(I \cap J)=R[x]$ since $p$ and $q$ are primes with $(p, q)=1$ and so $I \cap J=0$. But $\ell_{R[x]}(I)$ and $\ell_{R[x]}(J)$ do not contain constant. Therefore, $\ell_{R[x]}(I)+\ell_{R[x]}(J) \neq \ell_{R[x]}(I \cap J)$. So $R[x]$ is not a right IN-ring by Proposition 2.3. 
Recall that, a ring $R$ is called reduced ring if it has no nonzero nilpotent elements, a ring $R$ is called right p.p.-ring for all $a \in R, r_{R}(a)=e R$, where $e^{2}=e \in R$ and $R$ is called Baer ring, for all $I \leq_{R} R, r_{R}(I)=e R$, where $e^{2}=e \in R$.

As a result of Theorem 2.7, we can say the following corollary.

Corollary 2.9. Let $R[x]$ be a right IN-ring. Then $R$ is a right IN-ring in each of the following cases.

(1) $R^{2}=0$.

(2) $R$ is a reduced ring.

(3) $R$ is an Abelian (if every idempotent of $R$ is central) and von Neumann regular ring.

(4) $R$ is an Abelian right (left) p.p.-ring.

(5) $R$ is an Abelian Baer ring.

Proof. Assume $R[x]$ is a right IN-ring.

(1) By [1], if $R^{2}=0$, then $R$ is an Armendariz ring.

(2) By [2], reduced rings are Armendariz.

(3) Every Abelian von Neumann regular ring is a reduced ring.

(4) By [1, Theorem 6] or [7, Lemma 7], if $R$ is an Abelian right (left) p.p.-ring, then $R$ is an Armendariz (a Reduced and so Armendariz) ring.

(5) Every Abelian Baer ring is a reduced ring.

Hence $R$ is a right IN-ring by Theorem 2.7.

\section{Acknowledgments}

The author wishes to express his sincere gratitude to his Ph.D. supervisor Professor Abdullah Harmanci (Hacettepe University, Turkey) for his encouragement and direction. The author would like to thank the referee and Professor Yiqiang Zhou (Memorial University, Canada) for valuable comments and suggestions which improved the presentation of the paper.

\section{References}

[1] D. D. Anderson and V. Camillo, Armendariz rings and Gaussian rings, Communications in Algebra 26 (1998), no. 7, 2265-2272.

[2] E. P. Armendariz, A note on extensions of Baer and P.P.-rings, Journal of the Australian Mathematical Society, Series A 18 (1974), 470-473.

[3] M. Baser and A. Harmanci, Reduced and p.q.-bear modules, http://www.math.nthu.edu.tw/ $\sim$ tjm/myweb/FrameToAppear.htm.

[4] V. Camillo, W. K. Nicholson, and M. F. Yousif, Ikeda-Nakayama rings, Journal of Algebra 226 (2000), no. 2, 1001-1010.

[5] Y. Hirano, On annihilator ideals of a polynomial ring over a noncommutative ring, Journal of Pure and Applied Algebra 168 (2002), no. 1, 45-52.

[6] M. Ikeda and T. Nakayama, On some characteristic properties of quasi-Frobenius and regular rings, Proceedings of the American Mathematical Society 5 (1954), no. 1, 15-19.

[7] N. K. Kim and Y. Lee, Armendariz rings and reduced rings, Journal of Algebra 223 (2000), no. 2, 477-488.

[8] T.-K. Lee and Y. Zhou, Armendariz and reduced rings, Communications in Algebra 32 (2004), no. $6,2287-2299$. 
[9] _ Reduced modules, Rings, Modules, Algebras, and Abelian Groups, Lecture Notes in Pure and Appl. Math., vol. 236, Marcel Dekker, New York, 2004, pp. 365-377.

[10] R. Wisbauer, M. F. Yousif, and Y. Zhou, Ikeda-Nakayama modules, Beiträge zur Algebra und Geometrie 43 (2002), no. 1, 111-119.

M. Tamer Koşan: Department of Mathematic, Faculty of Science-Literature, Kocatepe University, ANS Campus, Afyon 03200, Turkey

E-mail address: mtkosan@aku.edu.tr 


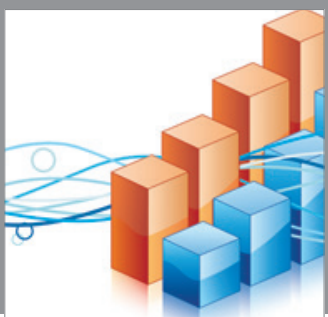

Advances in

Operations Research

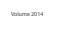

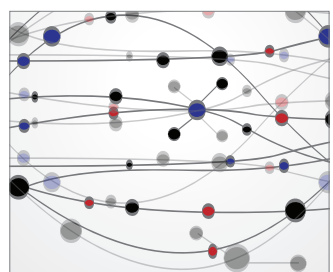

\section{The Scientific} World Journal
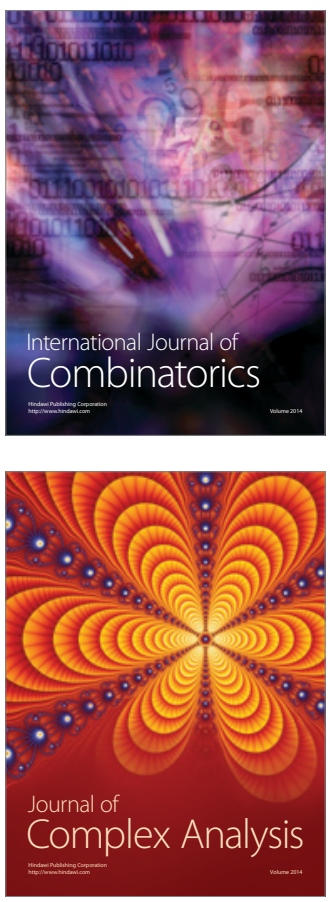

International Journal of

Mathematics and

Mathematical

Sciences
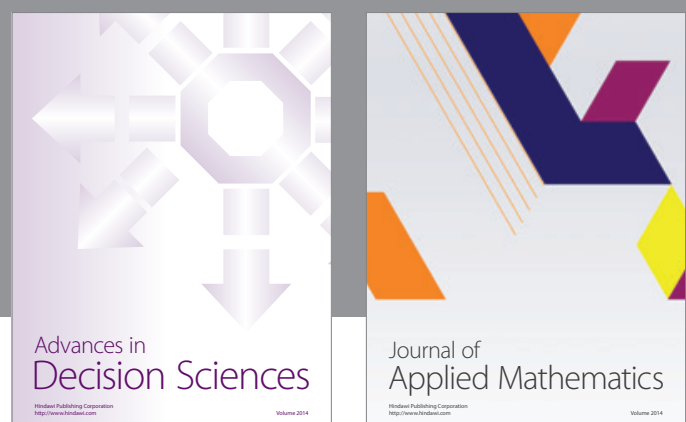

Journal of

Applied Mathematics
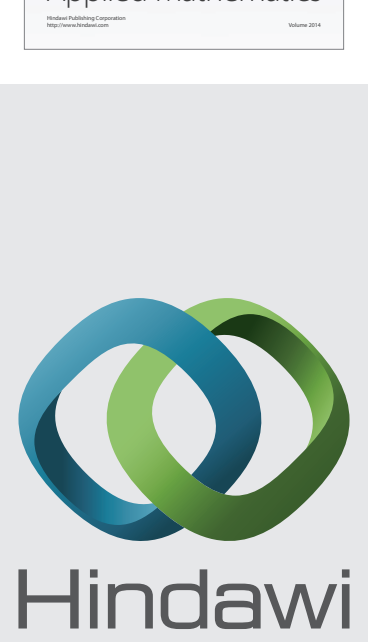

Submit your manuscripts at http://www.hindawi.com
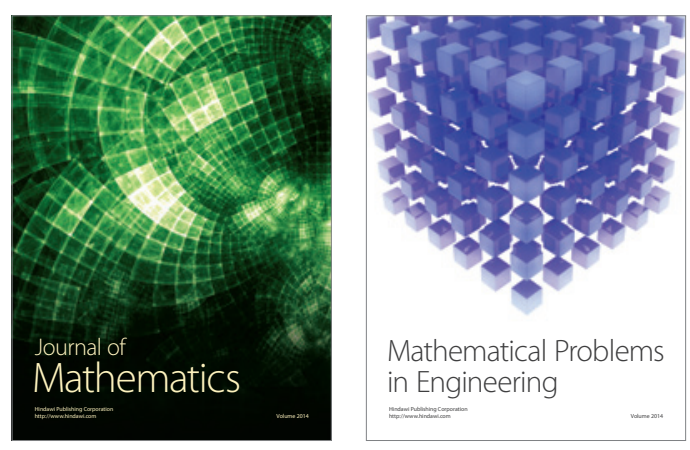

Mathematical Problems in Engineering
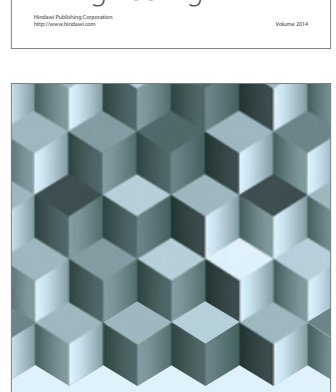

Journal of

Function Spaces
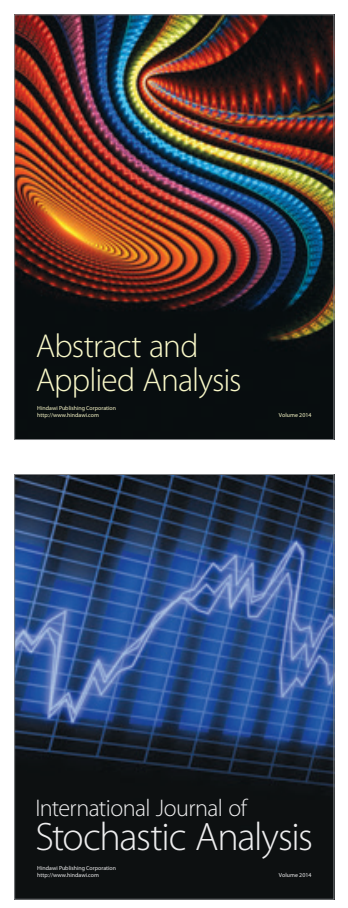

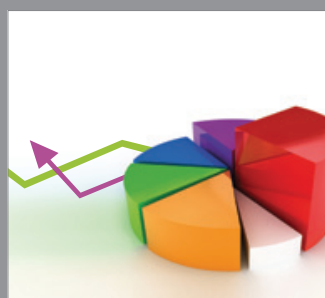

ournal of

Probability and Statistics

Promensencen
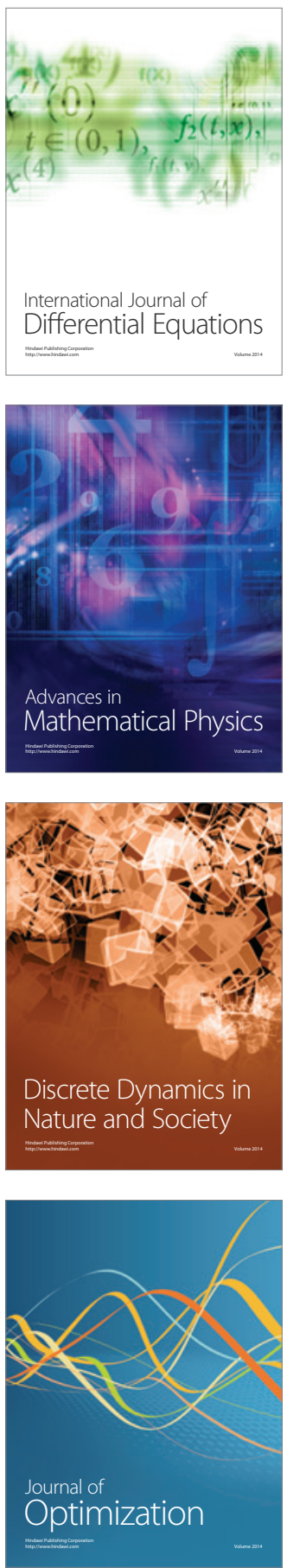